\title{
Inmersión, presencia y flow
}

\author{
Manuel Armenteros y Marta Fernández* \\ Universidad Carlos III-Madrid
}

Recibido: 14/10/2010

Aceptado: 29/11/2010

\begin{abstract}
Resumen: El objetivo de este trabajo es analizar el término 'inmersión' en los videojuegos para poder realizar una categorización posterior de los principales factores que intervienen, de qué manera influyen sobre el jugador y por qué unos juegos divierten más que otros. El artículo muestra que la inmersión es un estado transitorio con pérdida de consciencia temporal y espacial, que se produce en diferentes fases según el nivel de implicación del jugador, y que genera una satisfacción y experiencia positiva, determinada por factores humanos y factores tecnológicos. Asimismo, requiere que exista coincidencia entre las expectativas del jugador y el juego, consistencia entre la vida real y las convenciones del mundo representado, y que se produzca un impacto no trivial en las acciones del jugador.
\end{abstract}

Palabras clave: Inmersión / videojuegos / presencia / flow

\section{Immersion, presence and flow}

SUmmary: The paper analyzes the concept of immersion in video games in order to make a further categorization of the main factors involved, how they affect the player and why some videogames are more enjoyable than others. The article shows that 'immersion' is a transient state with loss of temporal and spatial awareness, which occurs in different phases depending on the level of player's involvement, and it generates an enjoyable, and positive experience, determined by human factors, and technological factors. It also requires a coincidence between the expectations of the player and the game, the consistency between real life and the conventions of the world represented, and a non-trivial impact on the player's actions.

Key words: immersion / videogames / presence / flow

* Agradecemos al profesor Francis Steen por sus comentarios y consejos útiles en el desarrollo de esta investigación. Agradecimiento especial a Jane Bitar por su gestión para obtener el acceso a las fuentes consultadas, así como su apoyo y atención permanentes. 


\section{Introducción}

1 nmersión' es un término que aparece en diferentes campos de estudio y su definición al día de hoy aparece de muy diversas formas. Originariamente, 'inmersión' es un término metafórico que deriva de la 'experiencia física' de estar sumergido en el agua (Murray 1997, citado en McMahan 2003: 68).

La mayoría de los autores consultados coinciden en que la 'inmersión' en un medio es una "sensación de alejamiento perceptivo del entorno real y de acercamiento a un entorno no real" (Jennet, Cox, Cairns, Dhoparee, Epps, Tijs y Walton 2008; Brown y Cairns 2004; Witmer y Singer 1998). Una "sensación" que hace olvidar al jugador que se encuentra sentado, frente al teclado o el joystick, y situado en un entorno que no es real pero que el jugador acepta como real.

McMahan (2003) y Brown y Cairns (2004) afirman en su análisis sobre el concepto de 'inmersión' que no está claro todavía qué es lo que motiva una definición u otra, y observan la necesidad de aclarar el término.

Para McMahan, las tres condiciones para conseguir la inmersión son:

1) Las "expectativas del usuario sobre el juego o el entorno" deben "coincidir o estar muy próximas al entorno del jugador".

2) Las "acciones del usuario" deben tener un "impacto no trivial" en el entorno.
3) Las "convenciones del mundo (representado)" deben ser "consistentes", aunque no coincidan con las convenciones del metaespacio.

Junto a las tres condiciones de McMahan que pueden conseguir la inmersión, un elemento más de los videojuegos que puede encontrarse como motivo de la sensación de inmersión, y que no tienen otros medios audiovisuales, como el cine, o impresos, como el libro, es la interactividad del videojuego, entendida como "forma de participar activamente en una narrativa" (Qin, Rau y Salvendy 2007). El juego exige una mayor participación en el usuario y presuponemos que una de las claves de la inmersión se encuentra en la forma en la que el jugador interactúa con el juego (gameplay).

Junto a los elementos citados anteriormente, existen otros como el grado de implicación y la satisfacción de juego producida en el jugador.

Brown y Cairns (2004: 1298), en un estudio cualitativo sobre cómo percibían la inmersión los jugadores de videojuegos, concluyeron que la inmersión es usada para medir el "grado de implicación" en el juego, el cual "se mueve a lo largo de un periodo temporal" y es "controlado por una serie de barreras". Estas barreras pueden ser eliminadas por una actividad humana, como la concentración, o ser eliminadas por otras, como el juego en sí mismo, por ejemplo aquellas barreras que dependen de la construcción del juego. 
Jennett, Cox, Cairns, Dhoparee, Epps, Tijs y Walton (2008), entre otros, relacionaron 'inmersión' con 'diversión' (enjoyment), y demostraron que la inmersión tenía una correlación con el grado de satisfacción producido. En esa misma línea, la investigación de Brown y Cairns (2004) reveló que ninguno de los que se sintieron inmersos afirmaron no haber disfrutado, o lo que es lo mismo, todos los que se habían sentido inmersos habían disfrutado de la experiencia.

Asimismo, el término 'inmersión' también aparece relacionado o como sinónimo de 'presencia', un concepto que aparece en los estudios de realidad virtual, y que a pesar de ser bastante similares, tienen diferencias significativas.

\section{Engagement, enjoyment y engrossment}

En la literatura consultada aparecen una serie de términos relacionados con el concepto de 'inmersión' como son involvement, engagement, enjoyment y engrossment. Se ha optado por buscar una palabra en castellano cuando esta proporciona un significado equivalente al término en inglés, como por ejemplo:

- 'implicación' por involvement

- 'compromiso' por engagement

- 'diversión' por enjoyment

- 'absorción' por engrossment
La mayoría de los autores consultados coinciden en describir la 'inmersión' como una 'sensación' en la que se produce una falta de consciencia temporal e incluso espacial. Jennett et al. (2008) describen esta experiencia como una "experiencia positiva" en la que el jugador pierde la "falta de consciencia del tiempo" y "de estar en el mundo real". Asimismo, es una experiencia que genera un "estado transitorio" que puede durar varios minutos.

Brown y Cairns (2004) realizaron un estudio cualitativo en el cual entrevistaron a siete jugadores sobre la experiencia de juego con el objetivo de desarrollar un concepto robusto de inmersión que pudiera usarse y contrastarse en otros contextos. En función de cómo fue esa experiencia, diferenciaron varios estados de implicación que identificaron como compromiso (engagement), absorción (engrossment) e inmersión total (total immersion).

\section{Compromiso (engagement)}

La primera fase, la fase de 'compromiso', viene determinada por las preferencias del jugador y por su nivel de destreza con el juego (Brown y Cairns 2004). Por ejemplo, si al jugador no le gustan los juegos deportivos, difícilmente llegará a sentirse implicado o comprometido con el juego.

El jugador ha de tener un grado de competencia sobre los controles del juego y superar una serie de barreras, como familiarizarse con la in- 
terfaz gráfica y los controles. Cuando un jugador no tiene experiencia de juego, está más atento en conocer el manejo de los mandos de control que en la propia dinámica del juego. Se produce una pérdida de implicación o compromiso hacia el juego en sí, en detrimento de un interés por conocer el manejo de la interfaz, como ocurre siempre que se desconoce el manejo de una herramienta o aplicación nueva. Una sensación similar a la que genera el conductor que coge el volante por primera vez para conducir, que presta más atención a la posición y manejo de los pies sobre el acelerador, el embrague, el freno y el cambio de marcha que en lo que ocurre delante. Debe existir, pues, una balanza entre nivel de habilidad del jugador y la oportunidad de juego. Si hay problemas de usabilidad y con los controles del juego no se podrá alcanzar el estado de compromiso ni experimentar otras sensaciones como la 'diversión' (enjoyment).

Carr (2002), citada en McMahan (2003), considera que el estado de compromiso en el jugador puede medirse. Carr utiliza el concepto 'deep play' como "capas de significado con valor estratégico que acumula el jugador" que permiten medir el grado de implicación del jugador con el juego. Por ejemplo, en un contexto de juegos de mesa como "Dragones y Mazmorras", el deep play vendría determinado por el grado de conocimiento de todos los monstruos y los diferentes trucos de magia.

\section{Absorción (engrossment)}

La segunda fase, previa a la 'inmersión total', es la fase de 'absorción' o estado de 'máxima atención'. Vendrá determinada por la construcción del juego o por las emociones que el juego genere en el jugador. Elementos expresivos como los gráficos y el sonido, o narrativos como la trama, pueden llamar la atención del jugador y pueden conseguir afectar sus emociones. Cuanto más atento y emocionado esté más inmerso se sentirá.

En este nivel, debido al tiempo, el esfuerzo y la atención que se le haya prestado, hay un gran nivel de inversión emocional en el juego. Esta inversión emocional provoca que el jugador quiera seguir jugando. El juego se convierte en la parte más importante de la atención de los jugadores y sus emociones se ven directamente afectadas por este.

\section{Inmersión total (total immersion)}

Un jugador alcanza un grado de 'inmersión total' cuando llega a ser competente en la interacción con el juego y es absorbido en las dinámicas del juego, o estas dinámicas tienen algunas inversiones emocionales en este (Reid, Geelhoed, Hull, Cater y Clayton 2005: 1733). Para Brown y Cairns (2004) la última fase que se puede alcanzar a lo largo de los diferentes estadios de implicación es la fase de inmersión total o presencia (presence). El jugador se siente "separado y desconectado de la realidad" hasta tal punto que el juego es lo 
único que le importa y tiene la "sensación de encontrarse físicamente en el entorno del juego" (Brown y Cairns 2004). Para estos autores, la inmersión total o presencia es una "experiencia efímera" controlada por las barreras de "atmósfera y empatía" y que están determinadas por "factores humanos, tecnológicos y contextuales".

\section{Presencia}

Hasta ahora se ha visto la 'inmersión' como una experiencia relacionada con el estado cognitivo del jugador. La 'presencia', según Witmer y Singer
(1998), se produce cuando "además del sistema cognitivo interviene el sistema perceptivo" para hacer sentir al individuo en un lugar donde físicamente no se encuentra, de ahí que haya sido estudiada principalmente en laboratorios de realidad virtual con simuladores y dispositivos casco-montados.

Para Witmer y Singer, la inmersión es "[...] un estado psicológico caracterizado por percibirse a sí mismo envuelto, incluido, e interactuando con un entorno que genera un continuo flujo de estímulos y experiencias" (1998: 3). En su investigación en el campo de los entornos virtuales con un total de 152

Tabla 1

Estados o fases de la inmersión a partir de la clasificación de Brown y Cairns (2004) y las aportaciones de otros autores

\begin{tabular}{|l|l|}
\hline Fase & Características \\
\hline Implicación (engagement) & $\begin{array}{l}\text { Determinado por: } \\
\text { las "preferencias del jugador" y por su "nivel de des- } \\
\text { treza" de la interfaz (Brown y Cairns 2004) } \\
\text { el "conocimiento del juego" o deep play (Carr, citada } \\
\text { en McMahan 2003) }\end{array}$ \\
\hline Absorción (engrossment) & $\begin{array}{l}\text { "Invisibilidad de los controles", "pérdida de conciencia } \\
\text { espacial" } \\
\text { Depende de la atención y emoción del jugador }\end{array}$ \\
\hline $\begin{array}{l}\text { Inmersión total (total immersion) } \\
\text { (Reid, Geelhoed y Hull 2005). }\end{array}$ & $\begin{array}{l}\text { (Reid, Geelhoed y Hull 2005: 1733). } \\
\text { El jugador es "competente con la interacción del jue- } \\
\text { go" ybsorbido en las dinámicas del juego" }\end{array}$ \\
\hline $\begin{array}{l}\text { Inmersión total o presencia } \\
\text { (Brown y Cairns 2004) }\end{array}$ & $\begin{array}{l}\text { - "Supera las barreras de atmósfera y empatía" } \\
\text { "Sensación de encontrarse físicamente en el entor- } \\
\text { no del juego" }\end{array}$ \\
\hline
\end{tabular}


individuos, a quienes se les pidió tareas como moverse a través de puertas $\mathrm{u}$ objetos en un entorno virtual (VE), los investigadores observaron una relación entre inmersión y presencia, de forma que un VE que genera una gran sensación de inmersión producirá una gran sensación de presencia.

Witmer y Singer (1998) tratan el término 'inmersión' dentro de una conceptualización de un término más amplio: 'presencia', asimismo, derivado, según la definición de Steuer (1992), citada en McMahan (2003), del término 'telepresencia', y que Lombard y Ditton (1997) engloban dentro de una de las seis dimensiones de presencia con el nombre de 'transportación'.

Para citar la definición de 'presencia' se utilizará la de Steuer (1992), transcrita literalmente como aparece en el texto de McMahan:

Presence is closely related to the phenomenon of distal attribution or externalization, which refer to the referencing of our perception to an external space beyond the limits of the sensory organs themselves. In unmediated perception, presence is taken for granted-what could one experience other than immediate physical surroundings? However, when perception is mediated by a communication technology, one is forced to perceive two separate environments simultaneously: the physical environment in which one is actually present, and the environment presented via the medium [...]. Telepresence is the extent to which one feels present in the mediated environment, rather than in the immediate physical environment. Telepresence is defined as the experience of presence in an environment by means of a communication medium. [...] In other words, "presence" refers to the natural perception of an environment, and "telepresence" refers to the mediated perception of an environment. This environment can be either a temporally or spatially distant "real" environment (for instance a distant space viewed through a video camera), or an animated but virtual world synthesized by a computer (for instance, the animated "world" created in a video game) (2003: 72).

[La presencia está estrechamente relacionada con el fenómeno de la atribución distal o externalización, que refiere a nuestra percepción de un espacio externo más allá de los límites de los propios órganos de los sentidos. ¿En la percepción no mediada, la presencia se da como hecho que es la experiencia del entorno físico inmediato? No obstante, cuando la percepción está mediada por una tecnología de comunicación, uno se ve obligado a percibir dos ambientes separados al mismo tiempo: el entorno físico, en el que uno está realmente presente, y el entorno presentado por el medio [...]. La telepresencia es el grado en el que uno se siente presente en el ambiente mediado, en lugar de en el entorno físico inmediato. La telepresencia se define como la experiencia de la presencia en un entorno a través de un medio de comunicación [...]. En otras palabras, la "presencia" se refiere a la percepción natural de un entorno y la "telepresencia" a la percepción mediada de ese entorno. Este entorno 
puede ser temporal o espacialmente distante del entorno "real" (por ejemplo, un lugar distante visto a través de una cámara de vídeo), o un mundo animado, pero un mundo virtual sintetizado por una computadora (por ejemplo, el "mundo" animado creado en un video juego) (2003: 72).

Como se puede leer en la definición de 'presencia', el autor distingue entre una presencia mediatizada o telepresencia y otra sin mediatizar. La telepresencia según Steuer originalmente significa la exitosa experiencia de presencia en un entorno teleoperado, como las operaciones en el espacio que dirigen los científicos desde la tierra utilizando dispositivos, mientras que la presencia, según Ryan (2001), significa el sentimiento que consiga "estar alli", en un espacio sintético, mientras que 'telepresencia' se reserva para situaciones 'teleoperadas', como operaciones en el espacio, intervenciones quirúrgicas, etcétera. Además, hace referencia a la percepción del espacio externo más allá de los límites de "los órganos sensoriales". Patrick, Cosgrove, Slavkovic, Rode, Verratti y Chiselko (2000), citados en Brown y Cairns (2004), especifican que la sensación de presencia viene dada por un "engaño" tanto al sistema perceptivo como cognitivo.

Pero ¿pueden los juegos generar sensación de presencia? Y ¿hay algún sentido por el cual a pesar de no estar "físicamente" provocado por un estímulo podamos tener la sensación de presencia?
Witmer y Singer (1998) planteaban estas preguntas ya por el año 1998 debido a que cuando aparecen las primeras definiciones de 'presencia', relacionadas con los entornos de realidad virtual, no existía el desarrollo de software y de representación visual que tienen actualmente los videojuegos. El término presence empezó aplicándose en el campo de los entornos virtuales tridimensionales, momento en el que los videojuegos estaban en su fase embrionaria y sus formas de representación visual no superaban las figuras pixeladas que se desplazaban de arriba abajo o de derecha a izquierda de la pantalla.

Esta idea parece estar relacionada con la conceptualización de 'presencia' como 'inmersión', y según estos autores un juego puede generar en el jugador un estado de inmersión pero no de presencia, mientras que si el jugador tiene la sensación de presencia forzosamente ha de tener también inmersión.

La investigadora McMahan (2003) propuso la posibilidad de utilizar en la inmersión a los videojuegos los criterios empleados para medir la presencia.

McMahan (2003) hace una distinción también entre ambas dimensiones: la perceptual y la cognitiva. Para McMahan, la presencia es "el resultado de una inmersión perceptual y de una inmersión psicológica". La inmersión perceptual es conseguida en la medida en que consigamos "bloquear" el mayor 
número de sentidos como sea posible del mundo exterior, y conseguir que el jugador solo perciba el mundo virtual que tiene delante, por ejemplo, haciendo uso de cascos, guantes hápticos, etcétera. La inmersión psicológica provendría de la absorción mental derivada de la experiencia en el mundo del juego.

McMahan cita el videojuego Myst como ejemplo donde se pueden observar ambas dimensiones: la perceptual y la cognitiva.

Debido a que Myst es un juego sobre el escritorio del ordenador, el grado de inmersión perceptual es limitado. El jugador está siempre limitado al tamaño relativo de la pantalla del ordenador y de la necesidad de usar el ratón [...] este nivel bajo de inmersión perceptual es superado, sin embargo, por un alto grado de inmersión psicológica (2003: 82).

La sensación de alejamiento del espacio real en el que se encuentra el jugador es un estado que puede conseguirse no solo con simuladores que activan los órganos perceptivos. La 'imaginación' es una forma de 'inmersión', que nos permite trasladarnos a espacios no reales como ocurre, por ejemplo, cuando se lee un cuento o vemos una película. De ahí que McMahan y Bucland (2002) hayan aplicado el concepto de 'presencia' a la sensación experimentada en el espectador de estar en el espacio de la película, ampliando el concepto de inmersión a inmersión diegética e inmersión no diegéti- ca, que aplican a los videojuegos. En la inmersión diegética el jugador es "capturado" en el mundo del juego, sus personajes, sus escenarios y su trama. La inmersión no diegética está relacionada con el interés que puede tener el jugador hacia todos aquellos elementos del juego que no pertenecen a la historia misma pero que lo "capturan" en el juego, como el deseo de conseguir puntos, sentirse ganador y mostrar a los otros jugadores su nivel de progreso.

Otros autores, como Patrick et al. (2000), citados en Brown y Cairns (2004), consideran, sin embargo, que los videojuegos y las películas no pueden proporcionar la experiencia de presencia.

\section{Inmersión y flow}

Csikszentmihalyi llevó a cabo varias investigaciones sobre experiencias que generaban satisfacción, y para ello entrevistó durante varios años a personas que dedicaban gran cantidad de tiempo y esfuerzo a actividades que eran difíciles pero que no recibían una recompensa económica, como escalar montañas o jugar al ajedrez. Csikszentmihalyi (1975), citado por Nacke y Lindley, describe flow como la "sensación holística que la gente siente cuando actúa con total implicación" (2008: 82). Flow es "[...] el estado en el que los individuos están tan implicados en una actividad que nada más parece importarles" (Csikszentmihalyi 1990, citado en Jennett et al. 2008). Es 
una experiencia tan gratificante que la gente está dispuesta sin importarles la dificultad o el peligro de dicha tarea (Csikszentmihalyi, citado por Sweeter y Wyeth 2005).

Para Brown y Carins (2004) el estado de flow es un "estado extremo de inmersión", una vez que han sido superados diferentes grados de implicación. Mientras flow es una experiencia óptima y por tanto extrema, la inmersión no es siempre tan extrema. En este sentido, la inmersión es precondición del estado de flow.

Brown y Carins (2004: 1300) comparan flow e inmersión, y observan que flow tiene paralelismos con el concepto de inmersión en tanto que "el estado de flow exige la atención, el sentido temporal se ve alterado y el sentido de uno mismo se pierde". También coinciden en cuanto al "[...] conocimiento y cualidades del jugador que exigen ambos estados". En cambio, "[...] la naturaleza fugaz de la inmersión total parece sugerir que es algo diferente a flow en este contexto".

Dieberger (2001) define flow como "el discurso interactivo que se produce en el transcurso del juego". Dieberger matiza que para que se dé continuidad debe generarse un continuo fluir sin interrupción, de manera que el jugador sienta plena libertad con lo que hace, aunque sea limitada. Otros autores consideran que ese continuo fluir sin interrupción debería conservar la atención del jugador el máximo de tiempo posible para mantenerlo concentrado. “El juego debería atrapar la atención del jugador rápidamente y mantenerla a través del juego $10 \mathrm{seg}$, $10 \mathrm{~min}, 10$ horas e incluso 100 horas de juego" (Pagulayan, Keeker, Wixon, Romero y Fuller 2003; Lazzaro 2004, citados por Sweetser y Wyeth 2005: 4).

Los ocho elementos importantes de flow comunes en la mayoría de la gente durante la experiencia óptima son (Csikszentmihalyi, 1990, citado por Cowley, Charles, Black y Hickey 2008: 2010):

- una tarea difícil pero que se pueda conseguir;

- se está completamente inmerso en la tarea, no importando otras preocupaciones;

- se siente plenamente el control;

- se siente la libertad completa de concentrarse en la tarea;

- la tarea tiene objetivos claros, sin ambigüedades;

- se recibe una retroalimentación inmediata en las acciones;

- $\quad$ se es menos consciente del paso del tiempo; $y$,

- el sentido de la identidad disminuye, pero se ve reforzada después.

En la lista de ocho elementos de flow se utiliza el término "inmersión" como un elemento, entre otros, necesario para adquirir la experiencia óptima o flow. Asimismo, Csikszentmihalyi utiliza para describir el estado de flow características del estado de inmersión como el control pleno de la tarea, me- 
nos consciente del paso del tiempo, objetivos claros y retroalimentación inmediata.

Entre las principales aportaciones de Csikszentmihalyi en el análisis de la "experiencia óptima" o flow existe la necesidad de establecer una tarea en los videojuegos que suponga un "reto para el individuo", quien debe tener una predisposición hacia tareas que requieran el uso de grandes habilidades que solo se alcanzan después de conseguir un nivel de experiencia, seguido de la práctica fuera del trabajo.

Un importante precursor de la experiencia de flow es la "conexión" entre las destrezas del individuo y el reto asociado con la tarea, en ambos casos sobre un determinado nivel (Csikszentmihalyi 1990, citado por Swetser y Wyeth 2005: 3).

Cowley, Charles, Black y Hickey et al. (2008) se preguntan si el estado de flow solo se experimenta mientras se persiguen determinadas actividades que requieran grandes habilidades, o y si lo necesario es que el sujeto haya invertido grandes esfuerzos en la formación para la actividad.

\section{Relación flow y enjoyment}

La correcta combinación de los ocho elementos de flow genera un sentido de gran satisfacción tan gratificante que la gente considera que gastar una gran cantidad de energía merece la pena simplemente para ser capaz de sentirlo (Csikszentmihalyi 1990, citado por Swetser y Wyeth 2005: 3).
Sweetser y Wyeth (2005) aplicaron los criterios utilizados para analizar la diversión al campo de los videojuegos, y desarrollaron el modelo gameflow que incluye ocho elementos: concentración, reto, destrezas del jugador, control, objetivos claros, respuesta, inmersión e interacción social.

En el análisis de dos juegos de estrategia en tiempo real, uno con un rating de calificación muy alto y otro bajo, encontraron que la concentración parecía ser un elemento particularmente importante para conseguir que el juego sea divertido. Los resultados de la investigación de Sweetser y Wyeth (2005) coinciden con la idea de Csikszentmihalyi de que para que el juego sea divertido requiere concentración y el jugador debe ser capaz de concentrarse en el juego.

Cuanta más concentración requiera una tarea, en términos de atención y carga de trabajo, más absorbente será. Cuando todas las destrezas de una persona son necesarias para hacer frente a los retos de una situación, la atención de esa persona estará completamente absorbida, y no queda energía salvo para cubrir dicha actividad (2005: 5).

La concentración, como un estado que se consigue tanto en la fase de inmersión como de flow, y como consecuencia de que se produzcan unos factores u otros, podría ser otra de las claves por las cuales un jugador se divierte jugando en un videojuego. 


\section{Conclusiones}

En el ámbito de los videojuegos, el concepto de 'inmersión' se refiere a un estado que experimenta el jugador $\mathrm{y}$ que:

- produce una pérdida de consciencia temporal y espacial;

- es transitoria (no dura todo el tiempo);

- genera en el jugador satisfacción y una experiencia positiva;

- viene determinada por una serie de factores humanos -como las preferencias del jugador, el nivel de destreza en el manejo de los mandos o el conocimiento de las dinámicas del juego- y tecnológicos, como la usabilidad, los problemas de control, el conocimiento de las dinámicas del juego o la construcción del juego;

- requiere que existan coincidencias entre las expectativas del jugador y el juego, y consistencia entre la vida real y las convenciones del mundo representado;

- es necesario que se produzca un impacto no trivial en las acciones del jugador; $y$

- se produce en diferentes fases según el nivel de implicación del jugador: compromiso (engagament), absorción (engrossment) e inmersión total (total immersion).

La inmersión forma parte de la presencia y del estado de flow, los cuales comparten características del estado de inmersión y generan una experiencia que va más allá de la que produce la inmersión.

En los estudios sobre realidad virtual, la inmersión es un estado previo a la presencia. La inmersión se refiere más a una dimensión psicológica, mientras que la presencia se refiere a una dimensión perceptual. La primera daría lugar a una inmersión psicológica y la segunda a una inmersión perceptual.

La inmersión también comparte características con el estado de flow o estado de experiencia óptima. Al igual que la inmersión, el estado de flow exige la atención, el sentido temporal se ve alterado y el sentido de uno mismo se pierde.

La experiencia óptima está relacionada con pasarlo bien (enyoyable). Una experiencia de juego será divertida (enjoyable) cuando se consiga capturar la máxima atención del jugador con tareas que requieran el máximo de atención y carga de trabajo.

\section{Bibliografía}

Brown, Emily y Paul Cairns (2004). "A grounded investigation of game immersion". CHI ACM Conference on Human Factors in Computing. Nueva York: ACM Press.

CARr, Diane (2002). "Playing with Lara", en King, Geoff y Tanya Krzywinska (eds.). ScreenPlay: 
Cinema/Videogames/Interfaces. Londres y Nueva York: Wallflower.

Cowley, Ben; Charles, Darryl; Black, Michaela y Ray Hickey (julio del 2008). "Toward an understanding of flow in video games". ACM Computers in Entertainment. Vol. 6, núm. 2.

Csikszentminalyi, Mihaly (1990). Flow: The psychology of optimal experience. Nueva York: Harper and Row.

- (1975). Beyond boredom and anxiety. San Francisco: Jossey-Bass Publishers.

Dieberger, Andreas (31 de marzo al 5 de abril del 2001). "Supporting narrative flow in presentation software". Conference on Human Factors in Computing Systems CHI ‘01. Seattle, Washington.

Jennett, Charlene; Cox, Anna L.; Cairns, Paul; Dhoparee, Samira; Epps, Andrew; Tiss, Tim y Alison WaLton (2008). "Measuring and defining the experience of immersion in games". International Journal of Human-Computer Studies. Vol. 66, núm. 9.

Lazzaro, Nicole. "Why we play games: Four keys to more emotion without story". <http://www.xeodesign. com/whyweplaygames/xeodesign_ whyweplaygames.pdf $>$. [Consulta: 3 de octubre del 2009].

Lombard, Matthew y Theresa B. Ditton (2000). "Measuring presence: A Literature-based approach to the development of a standarized paper-and-pencil instrument". 3rd Int. Workshop on Presence. Delft, Países Bajos.

McMahan, Alison (2003). “Immersion, engagement, and presence: A method for analyzing 3-D video games", en Wolf, Mark J. P. y Bernard Perron (eds.). The video game theory reader. Nueva York: Routledge.

McMahan, Alison y Warren Bucland (julio del 2005). "Cognitive schemas and virtual reality". Virtual Reality International, an affiliated Conference of the Human Computer Interaction (HCI) International Conference. Las Vegas.

NACKe, Lennart y Craig A. Lindley (3 al 5 de noviembre del 2008). Flow and immersion in first-person shooters: Measuring the player's gameplay experience. Congreso Future Play 2008. Toronto, Ontario, Canadá.

Pagulayan, Randy; Keeker, Kevin; Wixon, Dennis; Romero, Ramón y Thomas Fuller (2003). "Usercentered design in games", en Jacko, J. A. y A. Sears (eds.). The human-computer interaction handbook: Fundamentals, evolving techniques and emerging applications. Mahwah, Nueva Jersey: Lawrence Erlbaum Associates.

Patrick, Emilee; Cosgrove, Dennis; Slavkovic, Aleksandra; Rode, Jennifer A.; Verratti, Thom y Greg Chiselko (1 al 6 de abril del 2000). “Using a Large Projection Screen as an Alternative to Head- 
Mounted Displays for Virtual Environments". Congreso CHI.

QIn, Hua; RaU, Pei-Luen y Gavriel Salvendy (2007). "Player immersion in the computer game narrative". ICEC.

Ryan, Marie-L. (2002). Narrative as virtual reality: Immersion and Interactivity in Literature and Electronic Media. Baltimore M. D.: The Johns Hopkins University Press.

Steuer, Jonathan. "Defining virtual reality: Dimensions, deter- mining telepresence". Journal of Communication 42, núm. 4.

Sweeter, Penélope y Peta Wyeth (2005).

"GameFlow: a model for evaluating player enjoyment in games". ACM Computers in Entertainment. Vol. 3, núm. 3.

Witmer, Bob G. y Michael J. Singer (junio de 1998). "Measuring presence in virtual environments: A presence questionnaire". Presence. Vol. 7, núm. 3. Massachusetts: Massachusetts Institute of Technology. 